\title{
Desde el discurso médico: perspectivas sobre las disfunciones sexuales femeninas y masculinas en el Perú contemporáneo
}

\section{Skarlet Olivera}

solivera@pucp.edu.pe

Pontificia Universidad Católica del Perú (Lima, Perú)

(iD) https://orcid.org/0000-0002-2789-4759

\section{Rosario Ramos}

r.ramosc@pucp.edu.pe

Pontificia Universidad Católica del Perú (Lima, Perú)

(iD https://orcid.org/0000-0001-9784-3167

\author{
Alejandra Herrera \\ herrera.av@pucp.edu.pe \\ Pontificia Universidad Católica del Perú (Lima, Perú) \\ (iD) https://orcid.org/0000-0002-8964-5424
}

\begin{abstract}
Resumen
En sociedades históricamente heteropatriarcales como el Perú, el tema de la sexualidad se enmarca en un contexto de mitos, tabúes y desinformación, el cual es, en parte, construido por procesos de medicalización. Dentro de ello, las disfunciones sexuales son concebidas como trastornos o patologías que requieren ser diagnosticados y tratados por personal especializado. De este modo, la presente investigación se propone explicar las formas en que las disfunciones sexuales, tanto femeninas como masculinas, son abordadas actualmente desde el discurso médico. A través de una metodología eminentemente cualitativa que tiene como muestra a profesionales del campo de la salud, los principales hallazgos van referidos a las causas de las disfunciones sexuales y al enfoque de género presente en todo el proceso de diagnóstico, tratamiento y percepción. Como causa, el factor social juega un rol importante pero siempre circunscrito en lo que se considera psicológico o psicosomático. Asimismo, el aspecto generacional y de género en las personas entrevistadas es fundamental para explicar diferencias en torno a sus perspectivas médicas y heteronormativas. Finalmente, se concluye que, la percepción sobre disfunción sexual se ve afectada por construcciones de feminidades y masculinidades propias de sistemas hetero-cis-patriarcales.
\end{abstract}

\section{Palabras clave}

Disfunción sexual, medicalización, discurso médico, sexualidad, heteronormatividad

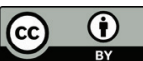




\title{
From the medical discourse: perspectives on female and male sexual dysfunctions in contemporary Peru
}

\section{Skarlet Olivera}

solivera@pucp.edu.pe

Pontificia Universidad Católica del Perú (Lima, Perú)

(iD https://orcid.org/0000-0002-2789-4759

\section{Rosario Ramos}

r.ramosc@pucp.edu.pe

Pontificia Universidad Católica del Perú (Lima, Perú)

(iD) https://orcid.org/0000-0001-9784-3167

\author{
Alejandra Herrera \\ herrera.av@pucp.edu.pe \\ Pontificia Universidad Católica del Perú (Lima, Perú) \\ (iD https://orcid.org/0000-0002-8964-54244
}

\begin{abstract}
Resumen
In historically heteropatriarchal societies such as Peru, the issue of sexuality is framed in a context of myths, taboos, and misinformation, which is, in part, constructed by processes of medicalization. Within this, sexual dysfunctions are conceived as disorders or pathologies that require diagnosis and treatment by specialized personnel. This research aims to explain the ways in which sexual dysfunctions, both female and male, are currently approached from the medical discourse. Through an eminently qualitative methodology that has health professionals as a sample, the main findings refer to the causes of sexual dysfunctions and the gender approach present in the entire process of diagnosis, treatment, and perception. As a cause, the social factor plays an important but always circumscribed role in what is considered psychological or psychosomatic. Likewise, the generational and gender aspect of the interviewees is essential to explain differences regarding their medical and heteronormative perspectives. Finally, it is concluded that the perception of sexual dysfunction is affected by constructions of femininity and masculinity typical of hetero-cis-patriarchal systems.
\end{abstract}

\section{Palabras clave}

Sexual dysfunction, medicalization, medical discourse, sexuality, heteronormativity 


\section{Introducción}

Como situación psicoafectiva biológica y disciplina basada en construcciones socioculturales, la sexualidad es parte vital de la identidad de los seres humanos. Por su naturaleza, las variaciones de un contexto a otro permiten que en torno a la sexualidad se constituyan problemáticas desde el nivel más individual e íntimo y, al mismo tiempo, que se vean plasmadas desigualdades y luchas de carácter estructural. En una sociedad históricamente heteropatriarcal como el Perú, el plano de la sexualidad también muestra estas desigualdades en temas como el género, que llevan a la persistencia -0 potencial crítica- de una serie de estereotipos o mitos. Dentro de la sexualidad, la cuestión del funcionamiento y disfunciones sexuales destaca como un área en la que las desigualdades y problemáticas se manifiestan abiertamente.

Sánchez et al. (2005, p. 76) definen las disfunciones sexuales como "trastornos psicosomáticos que impiden al individuo realizar el coito o gozar de él”. En el ámbito médico, la concepción contemporánea de salud sexual responde a un "estado de bienestar físico, mental y social en relación con la sexualidad, [...] posibilidad de tener experiencias sexuales placenteras y seguras, libres de toda coacción, discriminación y violencia" (OMS, 2020). En ese sentido, las disfunciones sexuales, como trastornos, deben ser diagnosticadas y tratadas, principalmente por profesionales de la salud.

Considerando que la medicalización ha sido una de las miradas preponderantes para abordar las disfunciones sexuales, la presente investigación enmarca el tema desde la perspectiva del discurso médico. Así, por medio de metodología cualitativa, el artículo busca responder la siguiente interrogante: ¿Cómo se aborda el problema de la disfunción sexual masculina y femenina desde el discurso médico o desde los procesos de medicalización en el Perú? A partir de ello, surgen otras interrogantes referidas a las diferencias entre disfunción sexual femenina y disfunción sexual masculina, y a las diferencias entre las perspectivas médicas tratadas. Una de las variables más importantes para explicar este último punto será el aspecto generacional.

A nivel latinoamericano, los estudios sobre disfunciones sexuales femeninas y masculinas han abordado la materia desde diferentes perspectivas y enfoques. Desde diagnósticos sobre la disfunción eréctil (Santibañez et al., 2016) hasta percepciones sobre la disposición en el uso del condón (Sánchez-Domínguez et al., 2010), la mayoría mantiene el marco de heteronormatividad para la construcción de argumentos y explicación de resultados. Algunas investigaciones como las de Mendieta (2018) y Mendoza y Ortiz (2009) salen de la matriz heteronormativa y apuntan a valoraciones y comprensiones más amplias. Sin embargo, el panorama de la región latinoamericana no se ve plasmado en el Perú: los estudios sobre disfunción sexual son sumamente escasos y ninguno cuenta con perspectiva de género, lo que permite que este estudio gane relevancia. Los análisis existentes se ajustan a la búsqueda de factores asociados y de prevalencia (Jiménez y Jara, 2014; Escajadillo, 2009), tanto para la disfunción sexual femenina como para la masculina. También omiten la perspectiva del discurso médico, que puede ser vital para tener una mejor aproximación y comprensión del fenómeno. Dada la naturaleza de la búsqueda de factores con significancia, las investigaciones son eminentemente cuantitativas y dejan de 
lado el enfoque cualitativo, que tiene la ventaja de tratar con una muestra pequeña, pero a profundidad.

El artículo se divide en seis secciones. En la primera parte, se explica cómo ha sido abordada la disfunción sexual femenina y masculina en Perú: mitos, tabúes, organizaciones, y avances y limitaciones en materia de investigación. En la segunda parte, se desarrolla el estado del arte junto con el marco teórico para presentar literatura con respecto a la medicalización de la sexualidad y de las disfunciones sexuales. En la tercera parte, se presenta la perspectiva de género en los procesos de medicalización. Posteriormente, en la cuarta parte, la sección metodológica detalla el tipo de estudio, la muestra y las herramientas de recolección de la información. En la quinta parte, se desarrollan los hallazgos, principalmente con base en la información obtenida a través de entrevistas semiestructuradas y en profundidad. Finalmente, en la sexta parte, tanto las conclusiones como las reflexiones finales enfatizan las diferencias encontradas.

\section{Disfunción sexual femenina y masculina en el Perú}

El tema de la sexualidad suele ser controversial en el Perú. Existen muchos tabúes, mitos y desinformación, los cuales generan conflicto en medio de valores y creencias aprendidas dentro de un sistema patriarcal dominante (Escajadillo, 2009). Esto constituye una red de desincentivos para que las personas que padecen disfunciones sexuales no recurran al apoyo de expertos. Como consecuencia, estos trastornos no llegan a ser detectados ni tratados a tiempo. Así, los tabúes y la desinformación impiden que las personas se manifiesten abiertamente sobre el tema, lo que genera un posible subreporte de casos de disfunción sexual. En esa línea, la actividad sexual generalmente no se registra en las historias clínicas, y la mayor parte de la investigación sobre sexualidad está referida a educación sexual (Escajadillo, 2009).

Respecto de centros u organizaciones sobre sexualidad, destacan tres organizaciones: "Psicólogo.pe", que es una red de psicólogas y psicólogos especialistas en sexología en Lima; APSES, la Asociación Peruana de Psicólogos Especialistas en Sexología; y Boston Medical Group, la principal clínica para disfunción sexual masculina en el país. La red de psicólogos y psicólogas cuenta con una página web abierta ${ }^{1}$ en la que personas interesadas en tener un tratamiento o diagnóstico pueden encontrar un listado de sexólogos, psicólogos y psicoterapeutas. La APSES es una organización civil de derecho privado que tiene como fin informar sobre los conceptos de la sexualidad humana y controlar la enseñanza de la educación sexual de acuerdo con lo establecido por la currícula del Ministerio de Educación (APSES, 2020). Por último, las clínicas para disfunción sexual masculina como Boston Medical Group cuentan con sedes en Lima, Arequipa y La Libertad, donde ofrecen servicios de diagnóstico y tratamiento a problemas sexuales masculinos. Entre los servicios ofrecidos, destaca que la atención para los clientes sea únicamente con "médicos hombres" (Boston Medical Group, 2020).

1 Véase https://www.psicologo.pe/sexologia 
Desde la academia, los escasos estudios sobre disfunción sexual femenina y masculina en el Perú abordan el tema de forma cuantitativa en relación con enfermedades cardiovasculares, cáncer, entre otras patologías. Los estudios más consolidados pertenecen a investigaciones hechas por estudiantes de la rama de salud de centros como la Universidad Cayetano Heredia, la Universidad Católica Sedes Sapientiae y la Facultad de Medicina de la Universidad Nacional de Piura (Escajadillo, 2009; Jiménez y Jara, 2014). Las investigaciones obtienen sus muestras de estudio mayormente de la zona norte del país, particularmente del sector urbano. Asimismo, el énfasis recae más en el estudio de la disfunción sexual femenina que en la masculina (Díaz-Vélez y Samalvides-Cuba, 2017; Escajadillo, 2009; Jiménez y Jara, 2014).

Escajadillo (2009) realiza un estudio para conocer la prevalencia de la disfunción sexual femenina (DSF) y los factores asociados en mujeres estudiantes de pregrado de la Universidad Nacional de Piura. Los hallazgos resaltan la prevalencia de disfunciones sexuales en casi el $40 \%$ de las estudiantes mujeres en la universidad. Uno de los principales alcances es que, además del trastorno orgásmico, el trastorno del deseo sexual también es bastante frecuente entre las mujeres con problemas de disfunción sexual. Sin embargo, como en todo estudio de corte cuantitativo, los factores asociados a la disfunción sexual -uso de anticonceptivos de emergencia, eyaculación precoz en la pareja y mala relación de pareja (Escajadillo, 2009) - no son profundizados ni se exploran los mecanismos causales.

Desde una perspectiva médica, Jiménez y Jara (2014) y Díaz-Vélez y Samalvides-Cuba (2017) se plantean determinar factores asociados a la disfunción sexual femenina (DSF). Las investigaciones recogen como muestra a pacientes atendidas en el Hospital Nacional Almanzor Aguinaga Asenjo en Chiclayo y en el Instituto Nacional de Enfermedades Neoplásicas. Se encuentra que el estado civil (ser casada, conviviente o viuda), tener ansiedad y los hábitos de fumar influyen en la disfunción sexual femenina. Estos factores, que se hallan dentro de características sociodemográficas, socioeconómicas y el estado de salud, entran en concordancia con los hallazgos de otras investigaciones realizadas en Chile y Estados Unidos (Díaz-Vélez y Samalvides-Cuba, 2017). En el caso específico de pacientes con cáncer, la diferencia recae entre mujeres con cáncer que han pasado por la mastectomía y aquellas que no (Jiménez y Jara, 2014). En ese sentido, uno de los principales aportes - con consenso- de los estudios mencionados recae en la comprensión de múltiples factores para determinar la presencia del trastorno en las pacientes.

En relación con las disfunciones sexuales masculinas, Santibañez et al. (2016) investigan cuantitativamente los factores asociados a la disfunción eréctil en pacientes con enfermedades cardiovasculares. Sabiendo que la disfunción eréctil (DE) afecta a millones de personas en todo el mundo y que puede ser un factor de riesgo para enfermedades cardiovasculares, los autores plantean tener como muestra de estudio a pacientes que están en rehabilitación cardíaca. El estudio abarca países como Chile, Brasil, Perú, Colombia, Argentina y Paraguay, y se resalta que el lugar de procedencia, la diabetes mellitus, la edad y el tabaquismo son factores asociados a la disfunción eréctil (Santibañez et al., 2016). No se han encontrado estudios académicos sobre disfunción sexual masculina centrados en el Perú. 
En suma, el tema de las disfunciones sexuales en Perú ha sido escasamente abordado. En su mayoría, las investigaciones son exploratorias y desde las ciencias de la salud. Ellas permiten generalizar entre los factores asociados, pero no profundizan de forma empírica ni desde otras disciplinas sobre la materia. Este último punto resulta particularmente relevante debido a que la salud no se encuentra aislada de las dinámicas histórico-sociales, políticas, culturales y económicas. La medicina social reconocía estos vínculos desde el siglo XIX². En la actualidad, la OMS reconoce la incidencia de dichos factores sobre la salud humana bajo el nombre de "determinantes sociales de la salud" (2009). Además, la sexualidad es uno de los pocos objetos de estudio que, desde las ciencias sociales, puede analizarse desde el nivel más íntimo hasta el nivel macrosocial. En relación con los centros de diagnóstico y tratamiento, el escaso número de estos (solo hay tres organismos a nivel nacional en total) no ayuda a aportar mayor visibilidad al tema y tampoco contrarresta la carga de prejuicios y estereotipos. Considerando lo mencionado, el estudio apunta a revisar el discurso médico desde el cual se abordan las disfunciones sexuales y, con ello, contribuir a investigaciones en ciencias sociales sobre el tema en el Perú.

\section{Medicalización de la sexualidad y de las disfunciones sexuales}

A nivel sociológico, el concepto de medicalización tiene su origen en el siglo XX como resultado de la influencia de dos tradiciones: la contracultura y la teoría del labeling (Murguía et al., 2016). Por un lado, desde la contracultura, se mostró que, a través de la medicalización, se buscaba controlar a los individuos sobre la base de problemas considerados "enfermedades mentales"3. Por otro lado, la teoría del labeling desarrolló todo un pensamiento crítico sobre cómo la salud había comenzado a etiquetar diferentes fenómenos como "desviaciones" (Murguía et al., 2016). Solo a partir de la década de 1990, la visión negativa hacia la medicina moderna es cuestionada (Murguía et al., 2016).

De acuerdo con la literatura revisada, la medicalización de la sexualidad ha sido estudiada desde tres campos en el siglo XXI: las humanidades; las ciencias sociales; $y$, de forma más interdisciplinaria, la medicina. En relación con las humanidades, desde la contracultura se ha permitido desarrollar una mejor comprensión de la medicalización como mecanismo de control sociopolítico. Este control plasma al individuo como un ser manejable, sin autonomía y que necesita depender de otros de forma permanente. Dentro de ello, el método genealógico foucaultiano ${ }^{4}$ ha permitido abordar el efecto de la medicalización en la consolidación de un marco heteronormativo (Cano, 2019; IguiñizRomero et al., 2018).

2 Por ejemplo, Engels, en The Condition of the Working Class in England, publicado originalmente en 1845, señalaba empíricamente relaciones entre la salud y fenómenos sociales producto de la explotación capitalista (la intensidad y condiciones de trabajo, el hacinamiento, la desnutrición, etc.). En la misma línea, Virchow, en 1848, en su informe sobre la epidemia del tifus en Silesia, señalaba cómo ciertas condiciones sociales pueden convertirse en causas de enfermedades.

3 Por ejemplo, en el primer volumen de Historia de la sexualidad, Michel Foucault (2009) presenta un análisis histórico sobre el control y la generación de discursos en torno a la sexualidad humana.

4 Hace referencia a la genealogía como forma de hacer historia al visibilizar la construcción de saberes y verdades (discurso). 
Desde las ciencias sociales, los trabajos han mostrado una diversidad de enfoques. En el caso de la ciencia política, el principal enfoque se halla en cómo los discursos estatales lograron generar una nueva representación de la sexualidad y los cuerpos a partir del énfasis en la medicalización (McManus y Mercado-Reyes, 2016; Pérez, 2020; Vallana, 2020). Desde la sociología, la representación de la medicalización ha sido abordada como instrumento de poder, en su interrelación con la variable de género y con las identidades fuera del espectro heteronormativo (Duran, 2017; Gavilán e Iriberri, 2014; Granados, Hernández y Olvera, 2017; Zuluaga, Cruz y Meneses, 2018). En la antropología, Simonetto (2016), a través de un estudio empírico con base en la obra "Sexuología" de Rinaldii Pelleginni, sostiene que la medicalización define lo que es normal y lo que no.

Finalmente, en el ámbito médico, la investigación en torno a la sexualidad humana ha mantenido su enfoque principalmente biológico: se ha centrado en la detección de factores asociados a cada trastorno en específico (como la disfunción eréctil, eyaculación precoz, etc.) (Jones y Gogna, 2012). También se ha abordado el rol de la sexología en el diagnóstico y tratamiento de las disfunciones (ibid.).

Desde la década del 2000, la búsqueda de los factores que conducen a que mujeres y varones no puedan llevar una plena vida sexual ha sido objeto de múltiples estudios (Hernández y Formental, 2007; Bravo et al., 2005; Sánchez et al., 2009; Sánchez et al., 2005; Sánchez et al., 2002a; Sánchez et al., 2002b). Aunque se ha mostrado interés por ambos sexos, la mayoría de estas investigaciones se han centrado en la disfunción sexual femenina, y han encontrado que las principales variables están relacionadas con 1) el género, 2) la carencia de educación sexual, 3) las experiencias sexuales traumáticas o abusos de infancia, y 4) el estilo de comunicación entre las parejas. Mucho de ello también está determinado por mitos y estereotipos impuestos por la sociedad e inclusive por no poder entender el sexo como una experiencia más allá del coito.

Así también, el aumento de las disfunciones sexuales y la búsqueda de soluciones ha generado gran oferta desde dos grupos: farmacéuticas y terapistas (ya sean sexólogos o psicólogos). Desde el campo médico también se ha evaluado si fármacos indicados para otros problemas -como la depresión- pueden servir para el tratamiento de algún tipo de disfunción sexual (Kingsberg y Woodard, 2015; Utian et al., 2004). En respuesta al uso y promoción de fármacos, se han escrito múltiples trabajos críticos a dicho enfoque, que señalan a la medicalización como una estrategia de las grandes farmacéuticas para continuar enriqueciéndose (Moynihan, 2004; Moynihan, 2005; Shankar y Subish, 2007; Zuluaga et al., 2018). Con relación a ello, especialistas como John Bancroft incluso discuten la existencia de ciertos trastornos como el del deseo sexual hipoactivo. Se sostiene que la reducción de interés sexual usualmente es una respuesta orgánica saludable y adaptativa frente a condiciones problemáticas en las relaciones o en la vida del individuo, por lo que no debería ser considerada una de las enfermedades que requieren tratamiento con fármacos (Moynihan, 2005).

Del mismo modo, Shankar y Subish (2007) exponen cómo existe una delgada línea entre la búsqueda por tratar un trastorno y ser un disease mongering. Este último, con la finalidad de vender el medicamento que ha generado, exagera respecto de los riesgos de la condición del paciente, así como de los beneficios de su producto. Otra respuesta 
al boom de los farmacéuticos han sido diversos estudios focalizados en el tratamiento de la terapia frente a disfunciones sexuales (Alcántara y Amuchástegui, 2004; Kaplan, 2002; Manzo y Yulis, 2004). Una de las críticas más importantes a esta vía ha sido el reduccionismo que aún existe en el momento de acudir a un terapista sexual, ya que existe una concentración en las conductas sexuales y se deja de lado las variables no sexuales que afectan directa e indirectamente en la sexualidad tanto de hombres como de mujeres (Manzo y Yulis, 2004).

\section{La perspectiva de género en los procesos de medicalización}

Varias de las investigaciones mencionadas previamente destacan el género como una de las variables que intervienen en los procesos sociohistóricos de medicalización en materia de sexualidad, pero no necesariamente se desarrollaron desde una perspectiva de género. En contraste, la investigación de Vallana (2020) profundiza en la construcción del saber y la práctica médica en ginecobstetricia. La autora encuentra que los significados construidos a partir de los discursos médicos sobre procesos fisiológicos femeninos contribuyeron a la reproducción de estereotipos de género en relación con la menstruación, la menopausia, el embarazo, el parto, etc. Con respecto a las disfunciones sexuales, Zuluaga et al. (2018) abordan los procesos de medicalización y de promoción de la enfermedad en sociedades occidentales contemporáneas a la luz del caso de las disfunciones sexuales femeninas. La "cultura farmacéutica" actual es señalada como uno de los aspectos que intervienen en la patologización de las disfunciones sexuales (primero, en la masculina y, posteriormente, en la femenina) al enlazar la vida privada de las personas con las dinámicas económicas y la política de producción farmacológica (Zuluaga et al., 2018). En otras palabras, la producción de fármacos se vincula a la "producción" de enfermedades y/o trastornos.

También se han realizado estudios considerando las experiencias sobre medicalización de la sexualidad en países determinados. En esa línea, Duran (2017) analiza cómo los modelos de género impuestos desde el discurso cientificista y liberal han sido parte de los procesos de medicalización y "cuestión social" en Chile y Argentina entre las décadas de 1860 y 1930. Desde una perspectiva más centrada en actores sociales (sin dejar de lado la dimensión estructural), Jones y Gogna (2012) desarrollan una investigación orientada a comprender cómo las y los sexólogos en Argentina perciben los procesos de medicalización de la sexualidad y si estos han incorporado en sus intervenciones una perspectiva de género. Encuentran que "la mayoría de los miembros del campo sexológico no visualizan los estereotipos de género, las asimetrías de poder entre varones y mujeres, y los mandatos sexistas como factores que intervienen en los problemas que su profesión intenta resolver" (Jones y Gogna, 2012, p. 55). Asimismo, el estudio de Hernandez y Formental (2007) constata que, entre pacientes de Centros de Salud Mental en Cuba, los estereotipos generados por las construcciones normativas sobre el acto sexual constituyen una barrera para el pleno disfrute de la sexualidad y para una mejor salud reproductiva.

En lo anterior, la variable de género es incorporada en términos binarios. Otras investigaciones han profundizado en procesos de medicalización que involucran a personas con identidades sexuales que exceden a la matriz heterocisnormativa preponderante. 
La investigación llevada a cabo por Granados, Hernández y Olvera (2017) analiza los procesos de medicalización y performatividad del género en su relación con la salud de un grupo de mujeres trans de la Ciudad de México. Butler (2007), desde la teoría queer, resaltaba cómo el género, además de ser una construcción social, posee un carácter performativo. Es decir, se crea una exigencia de vincular el sexo y el género a partir de las repeticiones de comportamientos y acciones, regulados a su vez por el disciplinamiento y el castigo: el género se construye mediante la performance repetitiva. En un contexto en el que la medicina contempla la transexualidad como una patología, los tratamientos se orientan a la "readecuación sexogenérica" de las pacientes, de modo que se "les impone la necesidad de autodenominarse bajo las categorías preestablecidas en el sistema sexogénero de base binaria" (Granados et al., 2017, p. 640). Otro estudio, desarrollado por Cano (2019), analiza la ginecología como una técnica de precarización o "prótesis de género" en tanto organiza y disciplina cuerpos a partir del sistema binario propio de la matriz heterocisnormativa de la sociedad en Argentina. Como consecuencia, desde esta especialidad médica se afectan principalmente a las identidades, corporalidades y modos de vida de personas LGTBIQ+.

En ese sentido, de acuerdo con los estudios presentados, se sostiene que son las variables no sexuales las que permiten explicar lo que sucede a nivel sexual en los individuos: lo no sexual está intrínsecamente relacionado a lo sexual, de ahí que las dinámicas económicas y las estructuras de género influyan en las formas en las que se entiende la salud y la sexualidad. La formación de profesionales de la salud no se encuentra exenta de estas influencias: el enfoque farmacológico predominante y las terapias sexuales reduccionistas terminan sistematizando la búsqueda de una solución al "trastorno" con tratamientos puramente biomédicos, relegando la importancia de los factores no sexuales ni biológicos sobre las disfunciones sexuales, como el sistema patriarcal, la falta de educación sexual integral, la carencia de herramientas emocionales entre las parejas, los traumas de infancia, entre otros. Por ello, el presente trabajo busca analizar el discurso médico con respecto a las disfunciones sexuales, ya sean masculinas o femeninas.

\section{Metodología}

Desde las formas de abordar la medicalización en los siglos XIX y XX hasta la intervención del género como variable sociohistórica, el tema de las disfunciones sexuales ha sido objeto de diferentes perspectivas y enfoques (tanto binarios como no binarios). Por consiguiente, con el fin de profundizar en el discurso médico y dar respuesta a la pregunta de investigación sobre la forma en que se abordan las disfunciones sexuales masculinas y femeninas desde los procesos de medicalización en el Perú, el estudio sigue una metodología cualitativa. Como sostienen Sampieri et al. (2003, p. 19), la metodología cualitativa permite aterrizar con mayor profundidad en el contexto, detalles y experiencias estudiadas. Para ello fue fundamental conocer las perspectivas y opiniones de personas vinculadas al campo de la sexualidad y disfunciones sexuales.

La investigación empleó los siguientes instrumentos de recolección de información: entrevistas semiestructuradas, entrevistas en profundidad y análisis de información 
secundaria. Las entrevistas en profundidad, que tienen por objetivo la comprensión de las perspectivas de los informantes en relación con sus vidas y experiencias, permitieron seguir "el modelo de una conversación entre iguales, y no de un intercambio formal de preguntas y respuestas" (Taylor y Bogdan, 1986, p. 3). La finalidad fue conocer y aproximarse a la perspectiva médica desde las propias palabras o desde el discurso de cada persona entrevistada. Para las entrevistas semiestructuradas, se elaboró una guía de preguntas con el fin de orientar la conversación. Asimismo, se tuvo en cuenta las fases de elaboración de reporte de investigación empírico propuestas por Pérez Serrano (1994) y Martínez Bonafé (1990): fase preactiva, interactiva y postactiva. Durante la fase preactiva, se seleccionó y delimitó el tema para posteriormente realizar la búsqueda de literatura para el estado del arte y marco teórico sobre los procesos de medicalización, la medicalización de las disfunciones sexuales y la perspectiva de género desde las disfunciones sexuales.

La fase interactiva abarcó el trabajo de campo. Para responder la pregunta de investigación, se tuvo una muestra de seis personas con formación en medicina humana, quienes participaron en entrevistas semiestructuradas y en profundidad. Utilizando un Protocolo de Consentimiento Informado, cuatro personas dieron su consentimiento para ser citadas de forma explícita y dos prefirieron permanecer anónimas ${ }^{5}$. Las cuatro personas entrevistadas no anónimas son las siguientes: Henry Romero (HR en adelante), de 62 años, médico ginecólogo; Antonio Grandez (AG en adelante), de 31 años, médico urólogo; Pablo Lucas (PL en adelante), de 32 años, médico urólogo; y Analucía Tejada (AT en adelante), de 22 ańos, estudiante de décimo segundo semestre de medicina humana. Las personas anónimas son dos: una médico ginecóloga de 33 años y una obstetra de 47. Se usaron las siguientes etiquetas para citar a las dos personas no anónimas: Entrevistada No1 y Entrevistada No2, respectivamente. La edad generacional también fue una variable importante, puesto que las perspectivas médicas tuvieron una connotación diferente entre las personas cuya edad se ubica en el intervalo 20-40 años y aquellas que se ubican en el intervalo 41-70 ańos.

La fase postactiva consistió en el análisis de las entrevistas realizadas y la elaboración del reporte final. En esta fase, la información recopilada fue analizada en relación con el marco teórico, la pregunta y los objetivos de la investigación. Finalmente, el documento final se elaboró haciendo énfasis en los hallazgos a partir de la información empírica.

\section{Análisis de los resultados}

La presente sección se aboca al análisis de la información recogida en las entrevistas a partir del estado del arte y marco teórico propuestos, con el objetivo de identificar elementos clave en los modos de abordaje de las disfunciones sexuales desde la medicalización. Para ello, el enfoque de género, y las construcciones normativas de feminidad y masculinidad de las sociedades heterocispatriarcales son considerados de manera transversal en el análisis. Este apartado se divide en cuatro subsecciones. Primero, las causas y diagnóstico de las disfunciones sexuales. Segundo, los tratamientos para las disfunciones sexuales.

5 De las seis personas entrevistadas, tres dieron su consentimiento informado de forma oral. 
Tercero, las perspectivas de los y las pacientes sobre el trastorno. La cuarta subsección está dedicada exclusivamente a las perspectivas médicas, aunque ello se encuentra en las tres subsecciones anteriores. Debe resaltarse que los hallazgos presentados a continuación no pretenden ser extrapolados al personal médico en general debido a las limitaciones de representatividad en la muestra. Por el contrario, se trata de presentar aportes iniciales, de corte exploratorio, que permitan el inicio de estudios más exhaustivos sobre la materia.

\subsection{Causas y diagnóstico de disfunciones sexuales}

A partir de las entrevistas realizadas y de la revisión de literatura previa, en el ámbito médico, se pueden entender las disfunciones sexuales desde dos perspectivas: 1) como un trastorno en el que la persona no refleja un "adecuado desempeño sexual" o 2) como un problema de salud que impide el "disfrute" del acto sexual (e incluso el acto sexual mismo). Desde la primera perspectiva, se prioriza el carácter de “objetividad” propio de los sistemas médicos occidentales o convencionales, regidos a partir del paradigma biomédico. En contraste, la perspectiva que define las disfunciones sexuales en torno a las dificultades en el goce de la sexualidad recupera el enfoque contemporáneo de la medicina convencional sobre la salud en general. La salud se entiende no como falta de "anormalidades" o patologías, sino como un estado de bienestar. Así, la dimensión subjetiva del paciente también puede ser incorporada en la dimensión de la salud, en este caso, asociada al disfrute pleno del acto sexual.

Para el diagnóstico, tanto en el caso de disfunciones sexuales tanto femeninas como masculinas, los especialistas y profesionales médicos se basan en la quinta edición del Manual de diagnóstico y estadisticas de trastornos mentales ${ }^{6}$. En este documento, las disfunciones sexuales están determinadas de acuerdo con la etapa del acto sexual que se encuentre afectada: deseo, excitación, orgasmo y/o resolución. En ese sentido, dentro de la categoría diagnóstica de "disfunciones sexuales", se encuentran los siguientes trastornos: eyaculación retardada, trastorno eréctil, trastorno orgásmico femenino, trastorno del interés/excitación sexual femenino, trastorno de dolor genito-pélvico/ penetración, trastorno de deseo sexual hipoactivo en el varón, eyaculación prematura (precoz), disfunción sexual inducida por sustancias/medicamentos, otra disfunción sexual especificada y disfunción sexual no especificada (Campo \& Herazo, 2018, p. 56).

Los procesos de medicalización cuentan con una dimensión normativa y simbólica que permite ejercer mecanismos de control sobre cuerpos e identidades. Además, pueden reproducir o potencialmente cuestionar el orden moral, cultural y sociopolítico en el que se encuentren enmarcados. En ese sentido, la medicalización de la sexualidad se legitima con su inclusión en clasificaciones internacionales de enfermedades, como la Clasificación Internacional de Enfermedades de la Organización Mundial de la Salud (OMS) y el Manual Diagnóstico y Estadístico de la American Psychiatric Association (APA) (Campo \& Herazo, 2018, p. 57).

6 Fuente: Entrevistada No 1, 2020. 
Las causas médicas de las disfunciones sexuales pueden estar asociadas a factores orgánicos, psicológicos o sociales. Entre los factores sociales, destacan las normas de género reproducidas mediante procesos de socialización. Sin embargo, el reconocimiento por parte de los profesionales entrevistados sobre la dimensión social como causa no fue homogéneo. De hecho, solo una persona entrevistada identificó los aspectos sociales como parte de las causas de las disfunciones sexuales de manera explícita. En las demás entrevistas, las y los profesionales o bien incorporaron estos aspectos dentro de los factores psicológicos (asociados a diagnósticos de depresión, estrés, entre otros) o bien no los mencionaron como parte de las causas de la enfermedad. Lo señalado por la única entrevistada que incorporó el enfoque contemporáneo en las disfunciones sexuales femeninas se presenta a continuación.

Muchas veces a las mujeres se las condiciona educativamente con el tema de la sexualidad y eso impide que, de alguna manera, puedan disfrutar su vida sexual de forma sana y plena. Y eso es lo que genera también una disfunción sexual así sean adultas, porque es una educación que han recibido desde pequeñas por parte tanto de sus familias como de la sociedad (Entrevista a AT, 2020)

$\mathrm{Al}$ contrastar las disfunciones sexuales femeninas y masculinas a partir de las causas que las generan, se encontró un elemento relevante vinculado a la violencia de género. Las experiencias traumáticas en relación con el acto sexual o la violencia en este ámbito también son identificadas como una causa únicamente de las disfunciones sexuales femeninas, aunque se aborda como causa psicológica, no social ${ }^{7}$.

Tal y como se ha mencionado en otros estudios de la región, el que la violencia y experiencias traumáticas solo constituyan posibles causas entre las disfunciones sexuales femeninas -mas no masculinas- evidencia que son las mujeres quienes tienden a estar más expuestas a la violencia de género en el ejercicio de su sexualidad (Sánchez et al., 2005). Adicionalmente, el hecho de que la mayoría de personas entrevistadas -así como se muestra también en páginas web de clínicas relevantes a nivel mundial como Mayo Clinic (2019; 2020) - entiendan estas causas como factores psicológicos genera una individualización e invisibilización de los factores sociales. Una de las personas entrevistadas lo ilustra bien a continuación.

Los factores asociados a la disfunción sexual femenina son bien parecidos a los factores asociados a la disfunción sexual masculina, como las enfermedades coronarias, la diabetes, el estrés, factores hormonales y psicológicos (Entrevistada No 2, 2020)

En las entrevistas, los factores sociales sí estuvieron presentes, no solo bajo la categoría de factores psicológicos, sino también como variables que intervienen en ámbitos externos a las causas médicas de las disfunciones sexuales. Se reconoció la influencia de factores sociales en la búsqueda o no de apoyo profesional por parte de los pacientes, en la continuidad de los tratamientos, y en la manera en que los pacientes perciben su propia enfermedad. Esto se desarrollará con mayor profundidad en secciones posteriores.

7 Fuente: Entrevista a AT, 2020; Mayo Clinic, 2019. 


\subsection{Tratamiento de disfunciones sexuales}

Los tratamientos para las diversas disfunciones sexuales se realizan en función de las causas que las y los médicos correspondientes identifiquen, así como del diagnóstico. En ese sentido, cuando las causas se deben a factores orgánicos, el tratamiento mayoritario es de carácter farmacológico, lo que engloba los tratamientos hormonales (principalmente orientados a disfunciones sexuales femeninas). En estos casos y, en particular, para ciertas disfunciones sexuales masculinas, el tratamiento quirúrgico también se encuentra como una opción, aunque se suele aplicar únicamente cuando el tratamiento farmacológico no da resultados. En caso de que la disfunción se deba a factores psicológicos, las terapias psicosexuales a cargo de profesionales de la salud como psicólogos o sexólogos son el tratamiento recomendado. En las entrevistas realizadas, sin embargo, se dio un mayor énfasis a los tratamientos con fármacos. Como señalan algunos entrevistados,

Disfunción eréctil, eyaculación precoz, sobre todo relacionados (sic), existen tratamientos con pastillas como PDEi5 (viagra, cialis, vardenafilo); por otro lado, con respecto a eyaculación precoz utilizamos SRI (paroxetina, dapoxetina) (Entrevista a AG, 2020)

La atrofia vaginal se puede tratar. Hay cremas, óvulos de estrógeno; también se puede tratar con plaquetas. Así como se inyecta para revitalizar la piel del rostro, también se puede inyectar en la mucosa vaginal. $Y$ ese es un procedimiento en realidad sencillo, no doloroso, porque la mucosa vaginal con anestesia en gel o en crema, una buena anestesia, se tolera bien, no es incómodo (sic) la aplicación con plaquetas (Entrevista a HR, 2020)

En la medida que se han detectado trastornos psicológicos como causas de los problemas de disfunción sexual femenina y masculina, parte de los tratamientos se han basado en fármacos antidepresivos, ansiolíticos, entre otros. Nuevas investigaciones farmacológicas han demostrado que estos mismos tratamientos tienen efectos positivos directamente en la problemática de salud sexual (Wespes et al., 2006). Lo dicho por las personas entrevistadas se condice con lo descrito en investigaciones farmacológicas. Aquello se ilustra de la siguiente manera:

En cuanto a la parte psicógena, el paciente en muchos casos tiene estados de ansiedad, de estrés, que son la causa de este tipo de problema. Esto ya se maneja en base a (sic) la indicación del psicólogo o psiquiatra (Entrevista a PL, 2020)

\subsection{Perspectivas de los y las pacientes sobre la enfermedad}

Como se mencionó al inicio del análisis, las entrevistas realizadas señalaron la influencia de factores sociales sobre la generación y reproducción de tabúes y estereotipos en torno al acto sexual, así como en los niveles de visibilidad del problema en el país. De este modo, elementos constituyentes de la matriz heterocisnormativa, sustentada en una concepción binaria del género, conducen a dificultades iniciales para abordar el tema por parte de los y las pacientes en el momento de la consulta médica, e incluso dificultan la búsqueda de apoyo profesional. Las palabras de algunas personas entrevistadas en relación con la disfunción sexual masculina son ilustrativas al respecto. 
La percepción en hombres es desde el punto de vista social. Se considera más importante el qué dirán, ya que es un tema tabú en nuestra sociedad. Es por eso que, en la mayoría de los casos, los hombres que padecen de este tipo de trastorno sexual suelen ocultarlo sin darle la importancia médica debida (Entrevistada No 2, 2020)

Es una enfermedad que trae mucha vergüenza y dificultad para admitirla debido a diversos problemas coyunturales. En algunos países, existe mucho machismo relacionado a este tema (Entrevista a AG, 2020)

Con respecto a las mujeres, también se presentan dificultades en la búsqueda de apoyo médico. De acuerdo con los profesionales de salud entrevistados, las mujeres tienden a considerar que la disfunción sexual femenina no existe, y que la frigidez o dispareunia es una consecuencia de la menopausia que no tiene solución o que no es un problema relevante ${ }^{8}$. En cuanto a las consultas médicas, de igual modo se presentan dificultades para abordar el tema. Como se señala a continuación,

En realidad, las pacientes que llegan a consulta ginecológica no lo manifiestan como motivo de consulta. Vienen por otras molestias y ya cuando le preguntamos "¿ $¿$ Tienes esto o esto?", "Sí, doctor. Me incomoda, me arde..." (Entrevista a HR, 2020)

Todavía nos falta avanzar con el problema de machismo, porque no acude la paciente con su pareja para hablar de estos temas, hay muchos varones que no apoyan a sus parejas en reconocer que tiene una disfunción sexual (Entrevistada No 1, 2020)

La manera en que las y los pacientes perciben y entienden la disfunción sexual que se les diagnostica también se encuentra asociada a la estructura heterocisnormativa de la sociedad. Este vínculo tiene raíz en los constructos de género binarios consolidados en los procesos de socialización. Los hombres aprenden que su masculinidad está directamente relacionada a su capacidad sexual, entendida como el logro de placer pleno e impartir poder sobre la mujer a través del coito (Hardy y Jiménez, 2001). En ese sentido, la virilidad se mide a partir del funcionamiento del aparato reproductor masculino. Por ello, los hombres relacionan las disfunciones sexuales como una pérdida total de sus capacidades como seres humanos ${ }^{9}$. Una de las personas entrevistadas a continuación lo ejemplifica de la siguiente manera:

A los hombres, desde muy pequeños, tanto en las publicidades, en los programas televisivos, en lo que escuchamos diariamente, se les atribuye una exigencia en cuanto al desempeño sexual. Y prácticamente lo vuelven la base de su vida. Entonces, una alteración de este nivel para un hombre que ha sido criado o ha escuchado constantemente ideas sobre este tema, no lo pueden interpretar o no lo interpretan en su mayoría de manera adecuada, sino más bien como un motivo de vergüenza (Entrevista a AT, 2020)

En el caso de las mujeres, la perspectiva se mantiene y lo dicho por dos entrevistadas es ilustrativo al respecto.

8 Fuente: Entrevista a AG, 2020.

9 Fuente: Entrevista a Analucía Tejada, 2020. 
A veces no reconocen que tienen una disfunción sexual. Algunas creen que es normal tener dolor durante las relaciones sexuales. En cuanto a la falta de deseo sexual, varias mujeres lo aceptan como parte del envejecimiento y acuden a consulta por ayuda porque el marido sí continúa con el deseo sexual (Entrevistada No 1, 2020)

En el caso de una mujer que presentaba disfunción sexual, su mayor preocupación no era lograr la satisfacción personal, sino el miedo a que, al no complacer a su pareja, a su esposo, este la deje o se aburra de ella (Entrevista a AT, 2020)

Los extractos de las entrevistas presentadas visibilizan la división de género que existe en torno al rol sexual que cada uno cumple en el marco de una sociedad históricamente heteropatriarcal. En contextos como este, la feminidad normativa se asocia al amor y a la entrega, en relación con la imagen de María (Mateos Sillero, 2013). De acuerdo con Lagarde (2005), "las mujeres están socializadas en el amor (los varones lo están en el poder) y en el darse al otro" (citado en Mateos Sillero, 2013, p. 313), lo cual también se traduce al plano de la sexualidad. Ya que los hombres se presentan normativamente como seres que deben recibir placer a través de las relaciones sexuales, las mujeres -en línea con los constructos de feminidad- deben cumplir el papel de complacer a su pareja y esforzarse para que él quede satisfecho. Cuando uno de los dos no logra ejecutar a plenitud ese rol, como se muestra en los ejemplos anteriores, parte de su identidad se ve dañada.

Desde otro enfoque, y también relacionado con las estructuras normativas del género, la visibilidad en la existencia de disfunciones sexuales femeninas y sus tratamientos resulta bastante reciente. Una de las formas más recientes con las que el tema de la disfunción sexual (especialmente femenina) se vuelve público es la publicidad. Antes de la publicidad, la preocupación o la importancia sobre la existencia de la disfunción sexual femenina era escasa ${ }^{10}$.

En este sentido, algunas personas entrevistadas destacaron el rol de las redes sociales y de la publicidad en tanto han permitido un cuestionamiento sobre los tabúes y mitos construidos en torno a las disfunciones sexuales tanto masculinas como femeninas. No obstante, en otras entrevistas, se destacó con mayor énfasis el carácter nocivo de la reproducción de feminidades y masculinidades normativas a través de las redes sociales, los medios y la publicidad. Desde esta perspectiva, en lugar de cuestionar los tabúes y mitos relacionados a las disfunciones sexuales y al acto sexual en particular, los medios y la publicidad constituyen mecanismos de reproducción del orden sobre el género, con lo cual también se agravan los problemas de disfunciones sexuales. Uno de los entrevistados lo explica bien a continuación.

La publicidad y, en este caso, publicidad en cuanto a medicamentos, en algunos casos hace que muchos pacientes, sobre todo jóvenes, se vean angustiados, estresados por no cumplir ciertos estándares. Entre comillas, estándares "adecuados", pero que no son en verdad una patología propiamente dicha, sino que ellos se crean esa necesidad, este temor o esta ansiedad, al creerse o al pensar o al tratar de llegar a ciertas características que puedan estar en la publicidad o en los medios de comunicación o en redes sociales (Entrevista a PL, 2020)

10 Fuente: Entrevistada No 2, 2020. 


\subsection{Perspectivas médicas}

El discurso médico sobre las disfunciones sexuales no resulta completamente homogéneo: varía de acuerdo con aspectos generacionales y de género. Entre los y las entrevistadas, la estudiante y la ginecóloga mujer más joven tomaban con mayor consideración las formas contemporáneas de entender las disfunciones sexuales femeninas y masculinas, es decir, entendiendo las disfunciones con relación a la afectación del disfrute de los y las pacientes en lugar de un desempeńo "inadecuado". Asimismo, consideraban la dimensión social de las disfunciones tanto entre las causas como de manera transversal en la materia. Los demás médicos centraron sus respuestas en aspectos fisiológicos asociados a las disfunciones sexuales, aunque sí reconocieron factores psicológicos como causa y su relación con las construcciones normativas del género, pero con mucho menor énfasis ${ }^{11}$. Entre ellos, solo los médicos hombres sostuvieron la perspectiva del "desempeño no adecuado" para definir las disfunciones sexuales.

La heteronormatividad y la cisnormatividad fueron aspectos comunes y transversales presentes tanto en la mayoría de las entrevistas como en las páginas web de clínicas destacadas y en la misma categorización del manual de diagnóstico. El presupuesto general en el que se enmarca el abordaje médico frente a problemas de disfunciones sexuales contempla que el o la paciente es una persona heterosexual y cisgénero. Así, a lo largo de las entrevistas, en cada alusión a problemas de pareja asociados a las disfunciones sexuales masculinas o femeninas, las y los profesionales de la salud asumen que se habla de una pareja heterosexual. De hecho, también se encontró que parte de las causas psicológicas de disfunciones sexuales son relacionadas por los médicos especialistas —en particular de la generación de 41 a 60 años - al modelo de familia tradicional. Así lo manifiesta uno de los entrevistados:

Quizás influye mucho el tema de los problemas económicos porque el varón, por nuestra cultura, mayormente es el que sostiene el hogar, la familia. Quizás se siente abrumado por toda la carga familiar y económica, y eso puede ser un factor que influye en el caso del varón (Entrevista a HR, 2020)

De las mujeres, el factor hormonal es el principal y también, como te decía, la mujer en esta sociedad es la que más se encarga del hogar, de los hijos. Y cuando los hijos crecen y se van, sienten lo del síndrome del nido vacío, sienten la falta de los hijos. El esposo está en el trabajo y si por ahí está inquieto, ya se derrumba todo (Entrevista a HR, 2020)

Por último, solo en el caso de la estudiante de medicina humana -la entrevistada más joven del intervalo de 20 a 40 ańos- se hizo un reconocimiento explícito de que el abordaje médico, que implica tanto el diagnóstico como el tratamiento de la enfermedad, se genera en función al paciente, y que su identidad y orientación sexual deben ser tomadas en cuenta para ello. Así se presenta a continuación:

En realidad, los tratamientos de esta patología, así como de cualquier patología, se dirigen a solucionar los problemas del paciente según su estilo de vida. Y obviamente sin que les genere daño físico a ellos o a otras personas, o algún daño emocional. [...] En la parte del

11 Fuente: entrevistas a Henry Romero, Antonio Grandez, Pablo Lucas y Entrevistada No 2. 
tratamiento psicológico, que es un pilar esencial dentro del tratamiento de disfunción sexual, de la misma manera, si ellos mantienen relaciones sexuales con personas de su mismo sexo o de sexo distinto, no hay ningún problema. El enfoque siempre está dirigido a solucionar el problema del paciente (Entrevista a AT, 2020)

\section{Conclusiones}

La presente investigación tuvo como finalidad comprender cómo han sido abordadas las disfunciones sexuales femeninas y masculinas desde los procesos de medicalización en el Perú. Se encontró que la matriz heterocisnormativa presente en sociedades como la peruana influye tanto en el modo en que se abordan este tipo de disfunciones desde el enfoque médico como en la percepción de los y las pacientes sobre su diagnóstico. En ese sentido, y en concordancia con los hallazgos de otros estudios sobre medicalización de la sexualidad en la región, aspectos como la heteronormatividad y cisnormatividad están presentes en el discurso médico de las personas entrevistadas.

Además, la distancia generacional entre los profesionales médicos entrevistados y su género influyeron en el modo en que abordaron el trastorno de las disfunciones sexuales. Los factores sociales asociados -tanto a las disfunciones sexuales masculinas como femeninas- fueron identificados por los médicos como posibles causas, pero enmarcados en la categoría de "factores psicológicos". Esto puede generar una tendencia a individualizar e incluso invisibilizar la dimensión social del trastorno a través de los procesos de medicalización. En cuanto a las percepciones de los pacientes, estas también se ven afectadas por las construcciones de feminidades y masculinidades propias de sistemas heterocispatriarcales.

Dada la poca cantidad de estudios en el país que aborden la medicalización de las disfunciones sexuales desde una perspectiva de género, el presente estudio aporta una mirada cualitativa inicial sobre el tema, que puede servir de insumo para una posterior generación de conocimiento en la materia. La perspectiva del discurso médico también permite entender cómo las construcciones de género se plasman desde la desigualdad y heteronormatividad. Una de las principales limitaciones del estudio yace en la falta de representatividad de la muestra de profesionales entrevistados. De hecho, la mayoría ejerce su profesión en Lima, por lo que la generalización de los hallazgos no es metodológicamente factible. No obstante, la metodología cualitativa empleada y las consideraciones del aspecto generacional permiten ahondar en el análisis de la información construida a partir de las entrevistas. Además, considerar el enfoque de género permite contar con sustento teórico para el análisis de la muestra.

Dentro de la agenda de investigación, una posible línea por seguir es el estudio de las disfunciones sexuales, pero desde la perspectiva de las y los pacientes involucrados. Hacerlo desde un enfoque biográfico o de historias de vida contribuiría a entender mejor qué dinámicas y variables son asumidas por las y los pacientes. En las entrevistas, por el énfasis detectado en el uso de fármacos para el tratamiento de las disfunciones sexuales, otras posibles líneas por seguir serían la promoción de la enfermedad y la cultura farmacéutica, enmarcado el contexto sociohistórico y cultural del Perú. Como 
prácticas médicas, ambas están presentes en las sociedades occidentales contemporáneas y cuentan con vacíos de información en el campo de las ciencias sociales.

Las desigualdades de género características de sociedades heteropatriarcales como la nuestra están también presentes en el ejercicio de la sexualidad de las personas, así como en la propia perspectiva médica. Por ello, otra línea de investigación sugerida apunta al ámbito de la salud pública. Al ampliar la reflexión sobre los procesos de medicalización de la sexualidad a partir de una perspectiva de género no binaria y transversal, se podrían construir nuevas posibilidades para promover y garantizar la salud de las personas. Después de todo, la salud, más que la falta de enfermedad, es un estado de bienestar que abarca todos los ámbitos de la vida del ser humano: la dimensión física, psicológica, social y sexual.

\section{Referencias bibliográficas}

Alcántara, E., y Amuchástegui, A. (2004). Terapia sexual y normalización: significado del malestar sexual en mujeres y hombres diagnosticados con disfunción sexual. Revista de estudios de género: La ventana, 2(20), 157-194.

APSES. (2020). Asociación Peruana de Psicólogos Especialistas en Sexología. https:// apsesperu.com/

Boston Medical Group. (2020). Problemas sexuales. Boston Medical Group. https:// bostonmedical.com.pe/duda-salud-sexual/problemas-sexuales

Bravo, C. S., Carmona, F. M., Nieto, D. P., Meléndez, J. C., y Rivera, G. (2002). Disfunción sexual masculina y calidad de la comunicación marital. Un estudio comparativo. Perinatología y Reproducción Humana, 16(1), 16-25.

Butler, J. (2007). El género en disputa. El feminismo y la subversión de la identidad. Paidós.

Campo, A. y Herazo, E. (2018). Novedades, críticas y propuestas al DSM-5: el caso de las disfunciones sexuales, la disforia de género y los trastornos parafílicos. Revista Colombiana de Psiquiatría, 47(1), 56-64. http://www.scielo.org.co/pdf/rcp/ v47n1/0034-7450-rcp-47-01-00056.pdf

Cano, V. (2019). Sexual Imaginaries and Medical In/Attention: Gynecology as Device for Hetero-Cis-Normalization. Sexualidad, Saludy Sociedad, (33), 42-58. https://doi. org/10.1590/1984-6487.sess.2019.33.03.a

Díaz-Vélez, C., \& Samalvides-Cuba, F. (2017). Factores asociados a disfunción sexual en mujeres atendidas en un hospital nivel III, Chiclayo, Perú. Revista chilena de obstetriciay ginecologia, 82(4), 386-395. https://doi.org/10.4067/s0717-752620170004003686

Duran, M. (2017). Sexualidad, producción y trabajo en el discurso higienista y eugenésico en Chile y Argentina, 1860-1930. Revista Nomadías, 23, 31-52.

Escajadillo, N. (2009). Disfunción sexual femenina en estudiantes de pregrado de una universidad pública del norte del Perú: estudio de casos y controles [Tesis para optar el grado de médico cirujano]. Universidad Nacional de Piura. http://repositorio.unp.edu.pe/ bitstream/handle/UNP/921/MED-ESC-VAR-09.pdf?sequence=1\&isAllowed $=n$

Figueroa, R., Jara, D., Fuenzalida, A., del Prado, M., Flores, D., y Blumel, J. E. (2009). Prevalencia de disfunción sexual en mujeres climatéricas. Revista 
médica de Chile, 137(3), 345-350. https://www.scielo.cl/scielo.php?script=sci_ arttext\&pid=S0034-98872009000300004

Foucault, M. (2009). Historia de la sexualidad 1: La voluntad del saber. Siglo XXI Editores. Gavilán, E., y Iriberri, A. (2014). Medios de comunicación como agentes que facilitan la medicalización de la vida: el ejemplo de la andropausia. Revista de Comunicación y Salud, 4, 49-67. https://dialnet.unirioja.es/servlet/articulo? codigo=4936070

Granados, J., Hernández, P., y Olvera, O. (2017). Performatividad del género, medicalización y salud en mujeres transexuales en Ciudad de México. Salud Colectiva, 13(4), 633-646. https://www.scielosp.org/article/scol/2017.v13n4/633-646/

Hardy, E., \& Jiménez, A. L. (2001). Masculinidad y género. Revista cubana de salud pública, 27(2), 77-88. http://scielo.sld.cu/scielo.php?pid=S0864-34662001000200 001\&script=sci_abstract

Hernández, I. y Formental, S. (2007). Diferencia de género y su influencia en las disfunciones sexuales. Revista Internacional de Andrologia, 5(3), 233-237. https:// www.sciencedirect.com/science/article/abs/pii/S1698031X07740633

Iguiñiz-Romero, R., Lossio, J., y Robledo, P. (2018). Por el bien de la nación: discursos científicos en favor de la medicalización del parto en el Perú, 1900-1940. História, Ciências, Saúde-Manguinhos, 25(4). https:/www.scielo.br/scielo.php?pid=S010459702018000400943\&script=sci_arttext\&tlng=es

Jiménez Tejada, J. J., y Jara Rojas, A. N. (2014). Factores asociados a la disfunción sexual femenina en pacientes con cáncer de mama en un hospital especializado en Lima, Perú [Tesis para optar el grado de maestro en Epidemiología Clínica]. Universidad Católica Sede Sapientiae. http://repositorio.ucss.edu.pe/bitstream/handle/UCSS/125/JaraJimenez_tesis_bachiller_2015.pdf?sequence=8\&isAllowed $=y$

Jones, D., y Gogna, M. (2012). Sexología, medicalización y perspectiva de género en la Argentina contemporánea. Ciencia, Docencia y Tecnología, XXIII(45), 33-59. https:// www.redalyc.org/pdf/145/14525317002.pdf

Kaplan, H. S. (2002). La nueva terapia sexual: tratamiento activo de las disfunciones sexuales. Alianza.

Kingsberg, S., y Woodard, T. (2015). Disfunción sexual femenina. Con enfoque en la Disminución del Deseo. The American College of Obstetricians and Gynecologists, 477-486. https://journals.lww.com/greenjournal/Documents/Feb2015_Translation _ Kingsberg.pdf

Manzo, C., y Yulis, C. (2004). Actualizaciones en terapia sexual. Terapia Psicológica, 22(2), 193-203. https://www.redalyc.org/pdf/785/78522211.pdf

Martínez Bonafé, J. (1990). El estudio de casos en la investigación cualitativa. En J. B. Martínez Rodríguez, Hacia un enfoque interpretativo de la enseñanza. Granada: Servicio de Publicaciones de la Universidad de Granada.

Mateos Sillero, S. (2013). Construcción de la feminidad normativa y sujeto político. Investigaciones Feministas, 4, 297-321. https://revistas.ucm.es/index.php/INFE/ article/view/43894

Matute, V., Arévalo, C., y Espinoza, A. (2016). Estudio transversal: prevalencia de disfunción sexual femenina y factores asociados en pacientes del Hospital "José Carrasco 
Arteaga". Revista Médica Hospital José Carrasco Arteaga, 8(1): 19-24. http://dx.doi. org/10.14410/2016.8.1.ao.03

Mayo Clinic. (2019). Disfunción sexual femenina. Mayo Clinic. https://www.mayoclinic.org/es-es/diseases-conditions/female-sexual-dysfunction/symptoms-causes/syc20372549

Mayo Clinic. (2020). Disfunción eréctil. Mayo Clinic. https://www.mayoclinic.org/es-es/ diseases-conditions/erectile-dysfunction/symptoms-causes/syc-20355776

Mendieta, G. (2018). Percepción de cuerpo y corporalidad en hombres que ejercen prostitución viril en Guadalajara, México. Revista Ciência \& Saúde Coletiva, 23(5), 15411549. http://www.scielo.br/scielo.php?pid=S1413-81232018000501541\&script=sci_abstract\&tlng=s

Mendoza, J.C. y Ortiz, L. (2009). Factores asociados con el uso inconsistente de condón en hombres que tienen sexo con hombres de Ciudad Juárez. Revista Salud Pública, 11(5), 700-712. https://revistas.unal.edu.co/index.php/revsaludpublica/article/ view/37557

McManus, F., y Mercado-Reyes, A. (2016). Constructing publics, preventing diseases and medicalizing bodies: HIV, AIDS, and its visual cultures. Sexualidad, Salud y Sociedad, (24), 69-102.

Moynihan R. (2004). FDA panel rejects testosterone patch for women on safety grounds. BMJ (Clinical research ed.), 329(7479), 1363. https://doi.org/10.1136/ bmj.329.7479.1363

Moynihan, R. (2005). The marketing of a disease: female sexual dysfunction. British Medical Journal, (7484), 192-194. https://www.ncbi.nlm.nih.gov/pmc/articles/PMC545000/\#ref5

Murguía, A., Ordorika, T., y Guerrero, F. (2016). Consideraciones epistemológicas en torno a la medicalización en América Latina: balances y propuestas. Ludus Vitalis, XXIV(46), 99-128.

Organización Mundial de la Salud [OMS]. (2020). Salud sexual. Organización Mundial de la Salud (OMS). https://www.who.int/topics/sexual_health/es/

Organización Mundial de la Salud [OMS]. (2009). Subsanar las desigualdades en una generación. Alcanzar la equidad sanitaria actuando sobre los determinantes sociales de la salud. Informe final de la Comisión sobre Determinantes de la Salud. OMS

Pérez Serrano, G. (1994). Investigación Cualitativa. Retos e interrogantes. Técnica y análisis de los datos. Vol. II. La Muralla.

Pérez, J. O. (2020). "Mais Médicos": Discourses, Bodies, and the Biopolitics of Medical Internationalism. Sexualidad, Saludy Sociedad, (34), 4-24.

Pretto, A. (2011). Analizar las historias de vida: reflexiones metodológicas y epistemológicas. Tabula Rasa, (15), 171-194. http://www.scielo.org.co/pdf/tara/n15/n15a10.pdf

Sampieri, R., Fernández-Collado, C., y Baptista, P. (2003). Metodología de la investigación. McGraw-Hill.

Sánchez-Domínguez, M. S., Leyva-Flores, R., Caballero-García, M., e Infante-Xibille, C. (2010). Disposición a usar condón en localidades con alta movilidad po- 
blacional de México y Centroamérica. Migración y Desarrollo, 7(15), 155-178. http://www.scielo.org.mx/pdf/myd/v8n15/v8n15a5.pdf

Sánchez, C., Carreño, J., Martínez, S., y Gómez, M. (2005). Disfunciones sexuales femeninas y masculinas: comparación de género en una muestra de la Ciudad de México. Salud Mental, 28(4), 74-80. http://www.scielo.org.mx/pdf/sm/v28n4/0185-3325sm-28-04-74.pdf

Sánchez, C., Carreño, J., Martínez, S., y Aldana, E. (2002a). Disfunción sexual masculina y su relación con el rol de género y asertividad. Perinatol Reprod Hum, 16(3), 123-131. https://www.medigraphic.com/pdfs/inper/ip-2002/ip023d.pdf

Sánchez, C., Morales, F., Pimentel, D., Carreño, J., y Guerra, F. (2002b). Disfunción sexual masculina y calidad de la comunicación marital. Un estudio comparativo. Perinatol Reprod Hum, 16(1), 16-25. https://www.medigraphic.com/pdfs/inper/ip2002/ip021d.pdf

Santibáńez, C., Anchique, C., Herdy, A., Zeballos, C., González, G., Fernández, R., Araya, V., y Soto, P. (2016). Prevalencia de disfunción eréctil y factores asociados en pacientes con indicación de rehabilitación cardíaca. Revista Chilenade Cardiología,35(3), 216-221 https://scielo.conicyt.cl/scielo.php?script=sci_arttext\&pid=S0718-85602016000300002

Shankar, P., y Subish, P. (2007). Disease mongering. Singapore Med, 48(4), 275-280. https://pubmed.ncbi.nlm.nih.gov/17384871/

Simonetto, P. (2016). Del consultorio a la cama. Discurso, cultura visual, erótica y sexología en la Argentina. Sexualidad, Salud y Sociedad, (22), 103-128. https://ri.conicet.gov.ar/handle/11336/97457

Taylor, S. J., y Bogdan, R. (1986). Introducción: ir hacia la gente. En Introducción a los métodos cualitativos de investigación: la buisqueda de significados. Paidós.

Utian, W., Braunstein, G., Buster, J., Lucas, J., y Simon, J. (2004). Testosterone transdermal patch improved sexual activity and sexual desire in surgically menopausal women: results from two phase III studies. Annual Meeting of International Society for the Study of Women's Sexual Health. Atlanta.

Vallana, V. (2020). "La enfermedad normal": Aspectos históricos y políticos de la medicalización del parto. Sexualidad, Saludy Sociedad, (34), 90-107.

Vega, G., Flores, P. J., Ávila, J., Becerril, A., Vega, A. J., Camacho, N., y García, P. (2014). Factores biopsicosociales asociados a la disfunción sexual femenina en una población mexicana. Revista chilena de obstetricia y ginecología, 79(2), 81-85. https://www.scielo.cl/scielo.php?script=sci_arttext\&pid=S0717-75262014000200003

Wespes, E., Amar, E., Eardley, I., Giuliano, F., Hatzichristou, D., Hatzimouratidis, K., Montorsi, F. y Vardi Y. (2006). Disfunción sexual masculina: Disfunción eréctil y eyaculación precoz. European Urology, 49(5), 806-15.

Zuluaga, J. C., Cruz, J., y Meneses, E. (2018). Disfunción sexual femenina y promoción de la enfermedad: aproximación desde el análisis crítico del discurso. Revista Internacional de Filosofía y Teoría Social, 25(4), 220-237. http://doi.org/10.5281/zenodo.3931071 